\title{
3 Forschungsfragen und Hypothesen
}

In der vorliegenden Studie gilt es herauszufinden, welchen Einfluss die Einstellung, subjektive Norm, wahrgenommene Verhaltenskontrolle sowie (ernährungspsychologische) Faktoren, wie Food Neophobia (die Lebensmittel-Neophobie), Food Technology Neophobia (die Abneigung gegenüber neuartigen Lebensmitteltechnologien), Food Disgust (der Ekel gegenüber Lebensmitteln), Sensation Seeking (die Suche nach neuen und intensiven Erfahrungen), Sustainability Consciousness (das Nachhaltigkeitsbewusstsein), auf die Akzeptanz von Insekten als Nahrungsmitteln haben. Auf Grundlage des aktuellen Forschungsstandes ergaben sich folgende Forschungsfragen (F) und Hypothesen $(\mathbf{H})$ :

F1: Welche Zusammenhänge bestehen zwischen dem Geschlecht, Alter, Schulabschluss und der Akzeptanz von Insekten als Nahrungsmittel?

F2: In welchem Zusammenhang stehen die Einstellung, subjektive Norm, wahrgenommene Verhaltenskontrolle und die Akzeptanz von Insekten als Nahrungsmittel?

H2: Die Einstellung gegenüber Insekten als Nahrungsmittel, die subjektive Norm und die wahrgenommene Verhaltenskontrolle sagen die Akzeptanz von Insekten als Nahrungsmittel voraus.

F3: In welchem Zusammenhang stehen Food Neophobia, Food Technology Neophobia, Food Disgust und die Akzeptanz von Insekten als Nahrungsmittel?

H3.1: Je höher die Lebensmittel-Neophobie und die Abneigung gegenüber neuartigen Lebensmitteltechnologien, desto geringer die Akzeptanz von Insekten als Nahrungsmittel.

H3.2: Je höher der Ekel gegenüber Lebensmitteln, desto geringer die Akzeptanz von Insekten als Nahrungsmittel.

F4: In welchem Zusammenhang stehen Sensation Seeking, Sustainability Consciousness und die Akzeptanz von Insekten als Nahrungsmittel?

H4.1: Je höher die Suche nach neuen und intensiven Erfahrungen, desto höher die Akzeptanz von Insekten als Nahrungsmittel.

H4.2: Je höher das Nachhaltigkeitsbewusstsein, desto höher die Akzeptanz von Insekten als Nahrungsmittel. 